\title{
Information und Energie in der Physiologie
}

\author{
Wolf D. Keidel und Manfred Spreng \\ I. Physiologisches Institut der Universität Erlangen, Erlangen
}

\begin{abstract}
ABST'RACT: Information and energy in physiology. Research in sensory physiology proves the usefulness of describing distinct functions of sensory systems from the point of view of information processing, neglecting energetic (metabolic) processes, which may occur simultaneously. On the other hand, complex metabolic processes play an important role in sensory reception and sensory communication. Adaptation - in quite a few situations resulting in an actual gain of sensory information - is based upon interacting metabolic processes. It is conceivable that various enzyme systems, such as coenzyme $B$, involved in the building and destruction of a particular exitatory substance, e. g., acetylcholine and cholinesterase, influence the speed of these different metabolic processes within the sensory cells. It is even possible to separate the damage done to these processes by using an electrophysiological recording of combined action potentials on the auditory nerve and accounting for the time course of adaptation according to RANKE's adaptation theory. The human central nervous system selects the $100 \mathrm{bit} / \mathrm{sec}$ processed for conscious perception from the $10^{9} \mathrm{bit} / \mathrm{sec}$ offered from all sensory receptors in two principal ways: (1) "Specific auditory information" is modulated by "unspecific" information processed through the reticular formation of the brain stem; (2) descending fiber systems alter selectively the information flow on every level of the auditory pathway. The filtered information perceived, in turn triggers a set of inborn and learned behavioral responses, such as speech and motor reaction, altogether representing appromaximately $10^{7} \mathrm{bit} / \mathrm{sec}$. Metabolic processes possibly involved in this optimizing system are largely unknown.
\end{abstract}

\section{EINLEITUNG}

Die Gleichberechtigung und enge Verknüpfung der beiden Begriffe En e rgie und $I \mathrm{n} f$ orm a tio $\mathrm{n}$ scheint in keinem anderen Teilgebiet der Physiologie eine so dominierende Rolle zu spielen, wie in der Sinnesphysiologie. Dabei mußte die Ansicht einer Unmöglichkeit der Erfassung sensorischer Vorgänge endgültig fallengelassen werden, als SHANNON \& WEAVER (1949) bezüglich nachrichtentechnischer Fragen die Möglichkeit einer Quantifizierung der Information aufzeigten. Unter dem Begriff „Information" ist der negative duale Logarithmus einer Ereigniswahrscheinlichkeit zu verstehen. Das Ereignis kann hierbei durch Gruppierungen von Signalen zeitlicher Natur (etwa elektrische Impulse in einem Fernsehkabel oder Aktionspotentiale auf einem Axon) oder räumlicher Natur (etwa die Schwarz-Weiß-Verteilung auf einer beschriebenen Fläche oder die Anordnung von Purin- und Pyrimidinbasen in Nukleinsäuren) gegeben sein. Einfacher läßt sich bekanntlich der Informationsgehalt eines Ereignisses (zum Beispiel eines Symbols) in der Anzahl der Auswahlschritte (Ja-Nein-Entscheidun- 
gen) messen, welche ein Empfänger benötigt, um aus einer verabredeten Menge (beispielsweise von Symbolen) auszuwählen. Die Maßeinheit für einen solchen Auswahlschritt ist bekanntlich „bit", eine Abkürzung des angelsächsischen Begriffs „binary digit".

\section{SINNESORGANSYSTEM OHR}

Der Vorteil dieser Betrachtungsmöglichkeit, die zunächst bewußt alle Energieprozesse gegenüber der entscheidenden Informationsverarbeitung vernachlässigt, wird deutlich, wenn man die Kompliziertheit eines solchen informationsverarbeitenden Systems im Organismus näher betrachtet. Abbildung 1 zeigt das Sinnesorgansystem Ohr in Form von Blockschaltbildern. Diese Blockschaltbilder sind hier Symbole für eine ganze neurale Verarbeitungsebene mit etwa 30000 Neuronen.

Es ist zunächst - ohne die Informationsübertragung in einem solchen Einzelelement, geschweige denn dabei ablaufende Energieprozesse im einzelnen zu betrachten - möglich, die Funktion des Gesamtsystems in sehr vielen Einzelheiten zu verstehen. Wie ersichtlich, befindet sich in der Umgebung eine Schallquelle, von der aus der Schallreiz über das Mittelohr, welches hier als Antransportorgan bezeichnet ist, auf das Innenohr triff. Im Innenohr findet eine Transformation von mechanischer Reizung in

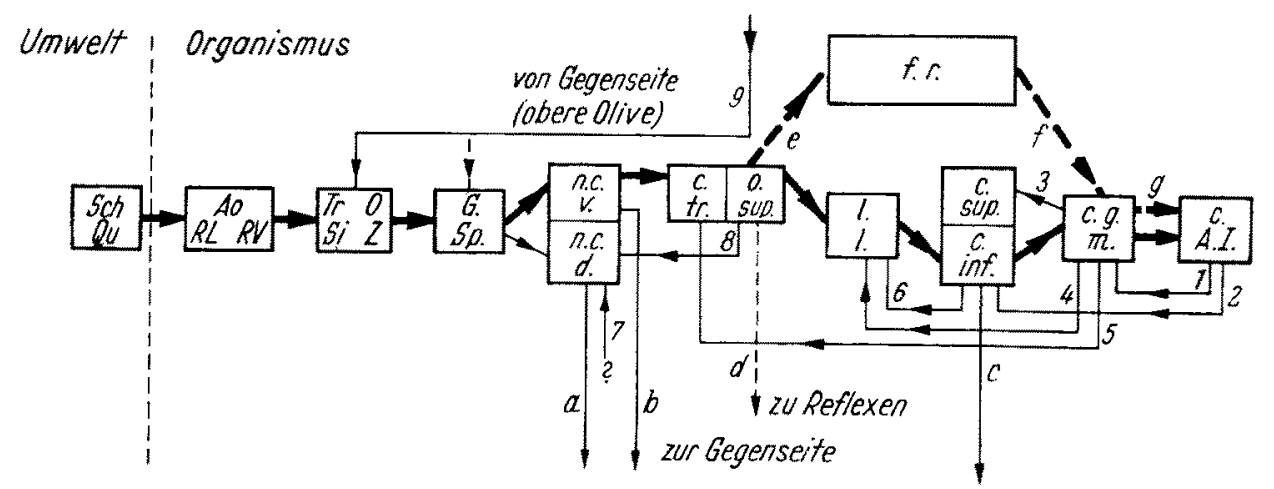

Abb. 1: Schaltung der Informationsverarbeitung beim Hören. „Spezifische Bahn“: kräfig ausgezogene Pfeile, Parallelmasche der ,unspezifischen Bahn“: kräfig gestrichelte Pfeile und zahlreiche Rückkoppelungsschleifen. SchQu: Schallquelle, Ao: Antransportorgan, RL: Reizleitung, RV: Reizverteilung, TrO: Transformationsorgan, SiZ: Sinneszelle, G.Sp.: Ganglion spirale, ncy: Nucleus cochlearis ventralis, ncd: Nucleus cochlearis dorsalis, ctr: Corpus trapezoides, o.sup.: Oliva superior, f.r.: Formatio reticularis, 1.1.: Lemniscus lateralis, c.sup.: Colliculus superior, c.inf.: Colliculus inferior, cgm: Corpus geniculatum mediale, cAI: Cortex area AI.

(Nach KeIDEL 1961b)

adäquate Reizung der abführenden Nerven statt. Die Hauptaufgabe dieser Transformation oder Wandlung fällt auf die Sinneszelle, und hier begegnet uns eigentlich das erste für Informationsverarbeitungsfragen wichtige Glied. Dessen Funktion soll im Laufe der vorliegenden Ausführungen (1) bezüglich der durch die Adaptation bereits hier stattfindenden aktiven Informationsverarbeitungsprozesse und (2) bezüglich der dabei ablaufenden Energie- und Stoffwechselvorgänge etwas näher beleuchtet werden. 
Der die Sinneszelle verlassende Nerv führt die durch den Reiz ausgelöste Erregung in pulsintervall-codierter Form zur nächsten Verarbeitungsebene (hier dem Cochleariskern), wo im allgemeinen Bahnungs-Hemmungsvorgänge zur Kontrastbildung, welche ja letztlich als Grundlage des Frequenzunterscheidungsvermögens dient, beitragen. Die Aufgaben der verschiedenen anderen Ebenen sollen in diesem Zusammenhang nicht näher erläutert werden; es darf nur darauf aufmerksam gemacht werden, daß eine große Zahl von Vermaschungen zur Gegenseite vorliegt (das Richtungshören ist nur mit beiden Ohren möglich). Außerdem laufen von hierarchisch höher liegenden Ebenen efferente Bahnen zu tiefer liegenden neuralen Verarbeitungsschichten mit bestimmten Aufgaben, beispielsweise der, selektiv zu drosseln (vgl. KeIDEL 1965).

In Höhe der Hirnrinde im akustischen Projektionsrindenareal A I ist zunächst das Ende dieser aufsteigenden sogenannten spezifischen Bahn zu suchen. Interessant ist außerdem noch die Verzweigung dieser spezifischen zu einer unspezifischen Bahn über den stammesgeschichtlich alten Hirnteil, nämlich die Formatio reticularis. Diese unspezifische Bahn mündet in Höhe der Hirnrinde in die spezifische Bahn wieder ein. Als letzte für die Informationsverarbeitung wichtige Bemerkung zu dieser Abbildung soll noch erwähnt werden, daß die Verbindungen zwischen den einzelnen Kästchen, also

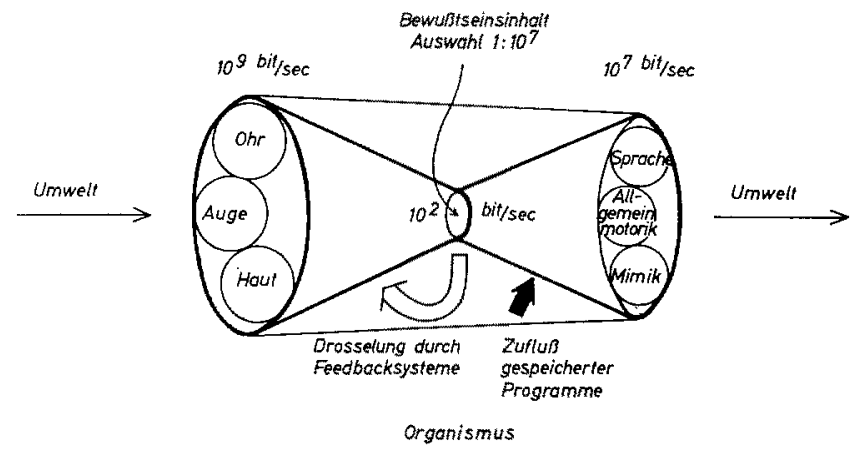

Abb. 2: Schema des Informationsflusses im Menschen. Die Optimalisierungsfunktion des ZNS sorgt dafür, daß die über Augen, Ohr und Haut aus der Umwelt zufließende Gesamtinformation bis zum Bewußtseinsinhalt auf den zehnmillionsten Teil selektiv gedrosselt wird. Hiervon werden wieder informationsreiche motorische Programme (Sprache, Mimik, Allgemeinmotorik) angestoßen, die zum Teil angeboren sind oder durch Lernvorgänge gespeichert werden. (Nach KerDet 1963b)

zwischen den einzelnen neuralen Schaltebenen, im Sinne einer Konvergenz-DivergenzSchaltung vorliegen, das heißt, es besteht keine einfache direkte neurale Durchverbindung, sondern es ist im allgemeinen ein. Element einer hierarchisch höheren Schicht mit einer Vielzahl einzelner Elemente der tiefer liegenden Schicht verbunden und umgekehrt (MountCASTLE 1959, KeIDEL 1961b).

Der Informationsfluß innerhalb eines solchen Sinnesorgansystems beziehungsweise der Informationsfluß, der auf die Gesamtheit aller Sinnesorgansysteme aus der Umwelt auftriff, ist größenordnungsmäßig mit $10^{9} \mathrm{bit} / \mathrm{sec}$ anzugeben (MILLER 1956, KüpFMÜLLER 1959). Der Organismus ist in der Lage, diesen Informationsfluß so zu reduzieren, daß vor einer bewußten Verarbeitung nur noch maximal etwa $100 \mathrm{bit} / \mathrm{sec}$ vor- 
liegen (Abb. 2). Diese extreme Reduktion um den Faktor $10^{7}$ fordert bestimmte opt im a lis ierende S y s te me, welche in der Lage sind, aus der Fülle der Informationen sinnvoll die für den Organismus lebenswichtige Information auszuwählen (KEIDEL 1963a). Derartige Systeme müssen insbesondere den Informationsfluß zwischen den verschiedenen Sinneskanälen kontrollieren können und die beschränkte Informationskapazität von $100 \mathrm{bit} / \mathrm{sec}$ jeweils de m Sinneskanal zuteilen, der im Augenblick die wichtigste Information anbringt. Für diese Aufgabe sind nicht nur die bereits

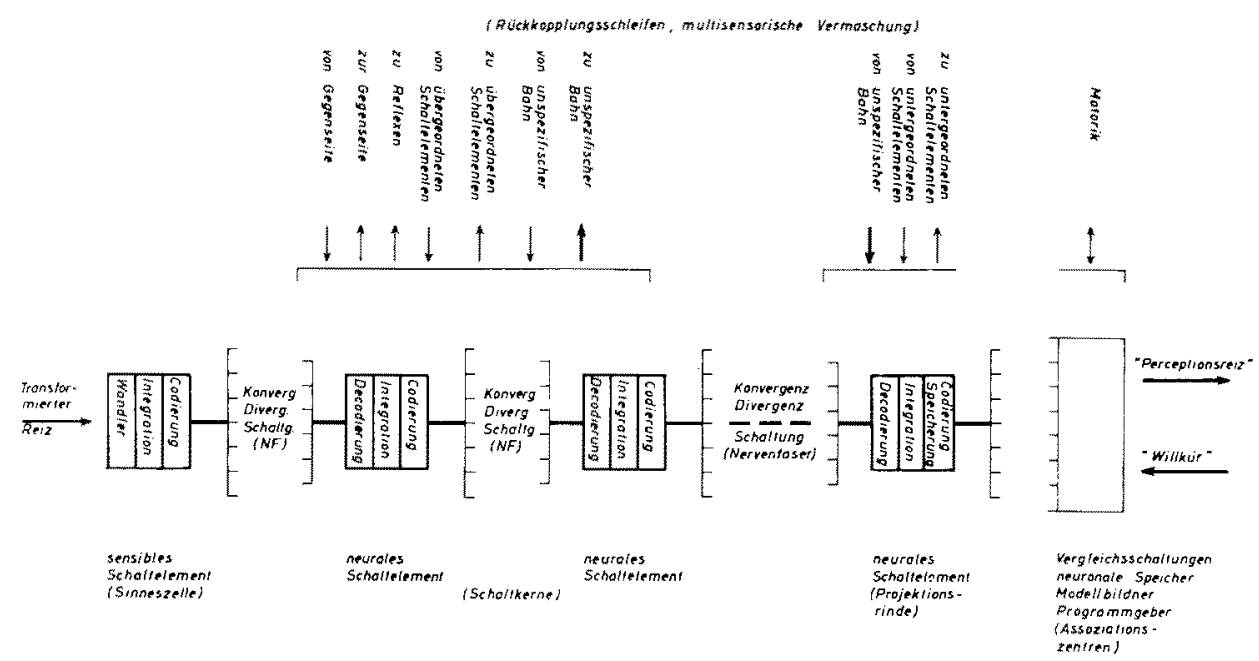

Abb. 3: Schematische Darstellung eines Sinnesorgansystems in Anlehnung an Abbildung 1. (Nadh SPRENG 1964)

erwähnten descendierenden und drosselnden Fasersysteme mitverantwortlich zu machen, sondern besonders auch die Verzweigung über die Formatio reticularis. Wie MAGOUN (1954) gezeigt hat, sind solche Verzweigungen nicht nur beim akustischen Sinneskanal gegeben, sondern jeder Sinneskanal (Haut, Auge) zeigt sowohl strukturell als auch funktionell Verzweigungen über das Stammhirn. Es besteht also die wohl begründete Vermutung, daß der Sitz einer solchen Optimalisierungsfunktion die Formatio reticularis ist, da sie ja Gelegenheit hat, mit jedem Sinneskanal zu korrespondieren und wahrscheinlich fähig ist, jeden Sinneskanal zu beeinflussen. Diese Beeinflussung ist aber nicht nur über descendierende Fasersysteme, sondern vor allem auch durch die unspezifischen Bahnen in Höhe der Firnrinde zu erwarten. Auch hierzu sind einige nähere Untersuchungen sowohl elektrophysiologischer als auch den Stoffwechsel betreffender Art anzustellen.

Kehren wir jedoch wieder zu dem in Abbildung 1 gezeigten Sinnesorgansystem Ohr zurück, nicht ohne jedoch mit Abbildung 3 eine noch schematischere Darstellung dieses informationsverarbeitenden Systems zu geben. Hier sind alle neuralen Schaltebenen als praktisch identische Funktionssysteme eingezeichnet, wobei diese Funktionssysteme jeweils aus einem Decodierungsteil, einem Integrationsteil und einem Codierungsteil bestehen. Im Decodierungsteil (den synaptischen Ubbergängen) werden die ankommenden pulsfrequenzmodulierten Signale in kontinuierlich amplitudenmodu- 
lierte Erregungen umgewandelt, im Integrationsteil (dem Komplex Neuron und Synapsen) wird ganz allgemein eine Zusammenfassung der verschiedenen Eingänge, also eine Informationsverarbeitung durchgeführt, schließlich wird an der Trennmembran des Neurons (zum abführenden Axon) eine erneute Codierung erfolgen.

In ganz ähnlicher Weise ist der Eingang dieser Informationsleitungskette, der Rezeptor, dargestellt, wobei der im allgemeinen als Decodierungsapparat bezeichnete

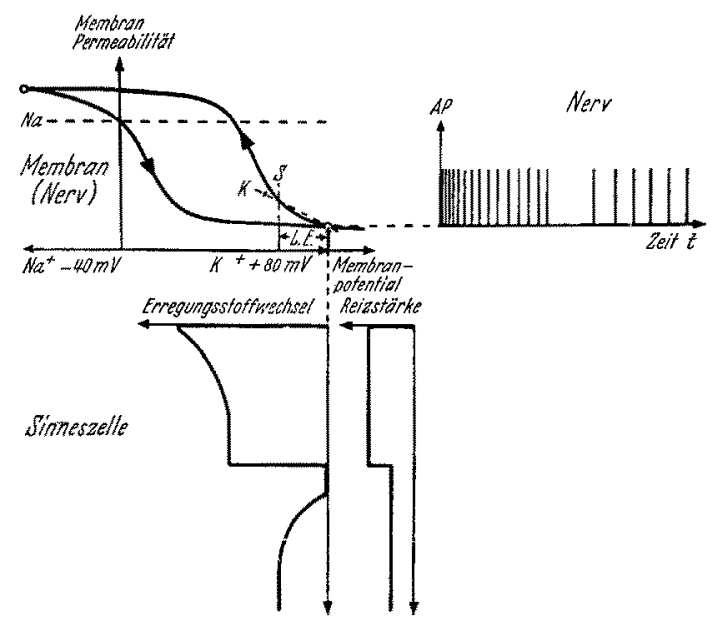

Abb. 4: Schema der Ubersetzung der Sinneszellerregung in die pulsintervallmodulierte Codeform der Nervenfaser durch die Grenzmembran zwischen Sinneszelle und Neurit. (Einzelheiten bei KerDeL 1961b)

Teil hier mit Wandler zu bezeichnen ist. Dieser Wandler hat die Eigenschaft, bestimmte Umweltreize, die als Lichteinwirkung, Schalleinwirkung und auch als mechanische Druckeinwirkung auftreten, mit Hilfe von Stoffwechselvorgängen in entsprechende körpereigene identische elektrische Erregungen umzusetzen.

Betrachten wir eine solche Umsetzung (Abb. 4), dann erkennen wir, daß ein Reiz, der hier in Form von Stufen aufgetragen ist (Zeitachse nach unten), nach Passieren des Wandlerteils dieser Sinneszelle die darüberliegende Erregungsform hervorruf, das sogenannte Generatorpotential. Dieses Generatorpotential wird über bestimmte funktionelle Eigenschaften der Trennmembran, welche mit der oben links schematisierten Membran-Kipp-Schwingungskennlinie (auf die nicht näher eingegangen werden soll) angedeutet sind, in die pulsintervallmodulierte Form umgesetzt und codiert weitergeleitet. Diese auf der Nervenfaser weitergeleitete Erregungsform ist ganz oben rechts dargestellt.

\section{ADAPTATION}

Unser besonderes Augenmerk wollen wir auf den Unterschied zwischen dem Reizsprung und der Erregung, die hinter dem Wandler auftritt, dem sogenannten Generatorpotential oder Rezeptorpotential (wobei zwischen beiden gelegentlich feine Un- 
terschiede gemacht werden [DAvIs 1961]) legen. Trotz eines einfachen Stufensprunges zeigt das Generatorpotential zunächst einen relativ steilen Anstieg auf einen hohen Wert, dann aber einen gewissen Abfall, bis ein Gleichgewichtswert erreicht wird. Diesen Vorgang bezeichnet man bekanntlich als Adaptation.

Diese Beziehung zwischen Eingangs- und Ausgangsgröße, die auf ein zusammengesetztes Proportional- und Differentialverhalten des Wandlers schließen läßt (Proportionalverhalten: einfaches Mitlaufen mit dem Reizsprung; Differentialverhalten: Erregung nur bei Anderung des Reizes; Addition ergibt den gezeigten Verlauf), ist in Abbildung 5 mit Registrierung nach MacNrcol (1956) am Limulus-Auge noch einmal deutlich zu erkennen.

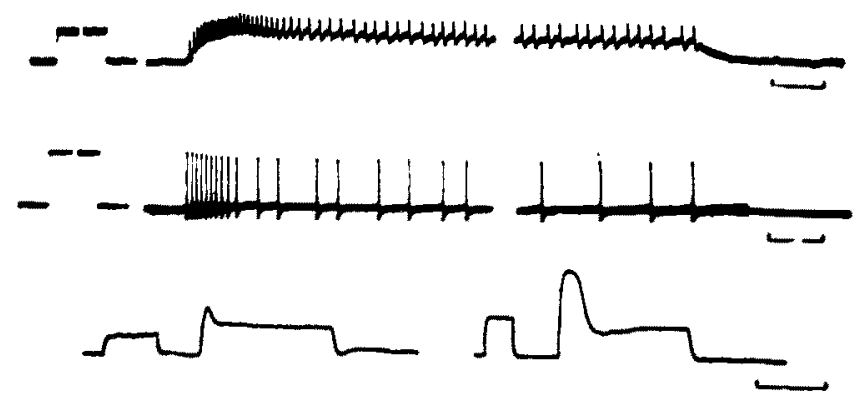

Abb. 5: Ubergangsfunktionen bei Leuchtdichtensprungreiz von oben nach unten an der Grenze zwischen Sinneszelle und Nervenfaser. (Nach MAcNicol 1956)

Die Adaptation, trotz Erregungsabnahme von einer Akkomodation der Nerven klar zu trennen, erweist sich nun, wie zahlreiche Untersuchungen zeigen, als ein außerordentlich wichtiges Funktionsglied bezüglich der Informationsverarbeitung in Sinnesorgansystemen (RANKE 1962). Diese bereits in der Sinneszelle ablaufenden Vorgänge ermöglichen dem Sinnesorgansystem eine Bereichseinstellung derart, daß jeweils der empfindlichste Meßbereich der mittleren Reizstärke angepaßt wird. Mit anderen Worten: schnelle Änderungen der Reizintensität bringen große Erregungsänderungen. Im dynamischen Fall ist also die Kennlinie zwischen Reizstärke und Erregung steil und demgemäß die Unterschiedsempfindlichkeit groß. Langsame Reizintensitätsschwankungen, welche ein praktisch proportionales Mitlaufen des adaptierten Zustandes ermöglichen, haben geringe Erregungsstufen zur Folge. In der Umgebung jeder Reizintensität, auf welche das Sinnesorgan adaptiert ist, werden schnelle Reizstärkeänderungen jedoch stets wieder durch maximale Erregungsstufen abgebildet. Sinnesorgansysteme sind also mit Hilfe der Adaptation in der Lage, so zu funktionieren, wie ein Meßinstrument mit unterdrückbarem Nullpunkt.

Es war vor allem Ranke (1962), der in jahrelanger Arbeit diese wichtige Eigenschaft der Adaptation klar erkannte. Er nimmt mit einer Gruppe anderer Autoren für das Zustandekommen dieser PD-Eigenschaften der Sinneszelle, also für das Zustandekommen des Adaptationsablaufes, eine Uberlagerung zeitlich verschobener Stoffwechselvorgänge an. Hierbei sind nicht die Konzentrationen, sondern die Reaktionsgeschwindigkeiten dieser Stoffwechselvorgänge das Korrelat für die elektrophysiologisch registrierbaren Erscheinungen. RANKE konnte zeigen, daß unter Zugrunde- 
legung allein des Massenwirkungsgesetzes die Erregungsvorgänge in der Sinneszelle zunächst für das Auge quantitativ beschrieben werden können. Die Lösung der von ihm formulierten Differentialgleichung - basierend auf der Überlagerung von zwei Stoffwechselvorgängen, einem Erregungsstoffwechsel- und einem Rückbildungsstoffwechselvorgang - ist in Abbildung 6 dargestellt. Mit dem aberschießenden Anteil und dem

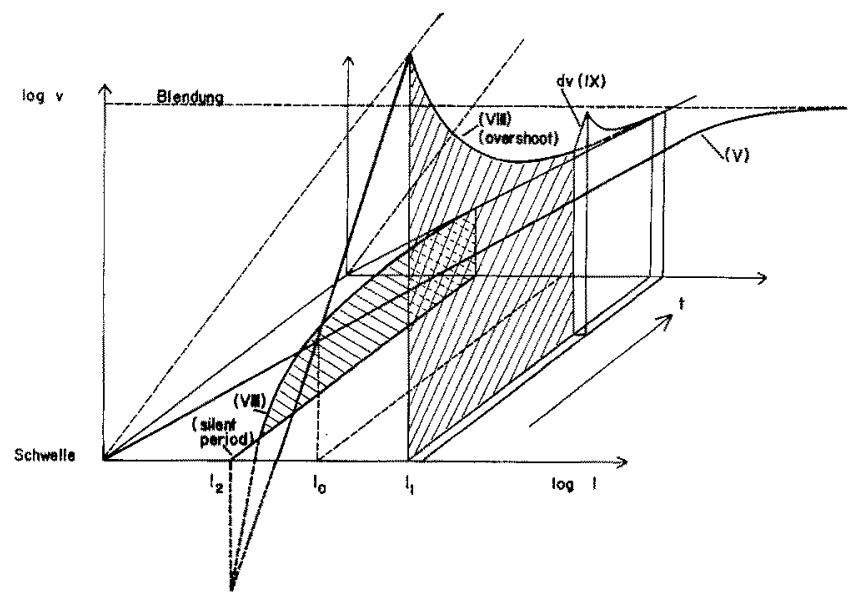

Abb. 6: Schema der Lösungen der Rankeschen Gleichungen für den Adaptationsvorgang in der Sinneszelle fußend auf dem Massenwirkungsgesetz. A b s z is s e : Logarithmus der Reizstärke I; O rd in a te : Logarithmus der Bildungsgeschwindigkeit des Erregungsstoffes; dritte Koordinate: Zeit. Die rechtsschrafferte Fläche läßt die Übergangsfunktion bei Reizsprung von $\mathrm{I}_{0}$ nach $\mathrm{I}_{1}$ mit initialem Overshoot und Abfall zum steady-state-Wert im Gleichgewichtszustand erkennen. Durch die linksschraffierte Fläche ist die Obergangsfunktion beim Reizabwärtssprung yon $I_{0}$ nach Ig mit silent period hervorgehoben. (Nach RANKE aus KEIDEL 1961a)

sich dann im Sinne eines Fließgleichgewichtes einstellenden Gleichgewichtsendwert stimmt der Verlauf der ermittelten Kennlinie mit den experimentellen Befunden sehr gut überein (KEIDEL 1961a, b, RANKE 1962).

Ist beim Auge zumindest das Vorhandensein von Stoffwechselvorgängen in det Sinneszelle bekannt, so fehlten jedoch bisher beim akustischen Sinnesorgansystem entsprechende experimentelle Beweise für den Einfluß von zwei oder gar mehreren Stoffwechselvorgängen auf die Adaptation. Die Ergebnisse einer in unserem Institut durchgeführten Untersuchungsreihe sollen im folgenden kurz skizziert werden (STANGE, SPRENG \& KeIDel 1964).

Nicht nur die einzelne Sinneszelle zeigt Adaptationsverhalten, sondern auch die Gesamtheit der erregten Sinneszellen bei Einwirkung eines Reizes. Abbildung 7 zeigt diesen Adaptationszeitgang, der am Summenaktionspotential des Akustikusnerven erkennbar ist. Dieses Summenaktionspotential stellt dabei das Integral über Ort und Zeit der einzelnen Erregungen, wie sie beispielsweise in Abbildung 5 gezeigt sind, dar. Es ist zu erketnen, daß der Reiz, in der unteren Bildhälfte in Form des Schalldruckes aufgezeichnet, konstant verläuft, während das Summenaktionspotential hinter der Sinneszelle deutliche Adaptation erkennen läßt. Das Transformations- oder Wandlerorgan adaptiert dabei um so mehr, je höher die pro Zeiteinheit angebotene Reizzahl (Recht- 
eckschalldruckstöße), wie sie unten dargestellt worden ist, zunimmt. Das bedeutet: Mit zunehmender Reizfrequenz wird der Gleichgewichtsendwert immer kleiner, und wir können die Größe des Adaptationszustandes erkennen, wenn wir jeweils den Anfangswert und den Gleichgewichtsendwert betrachten.

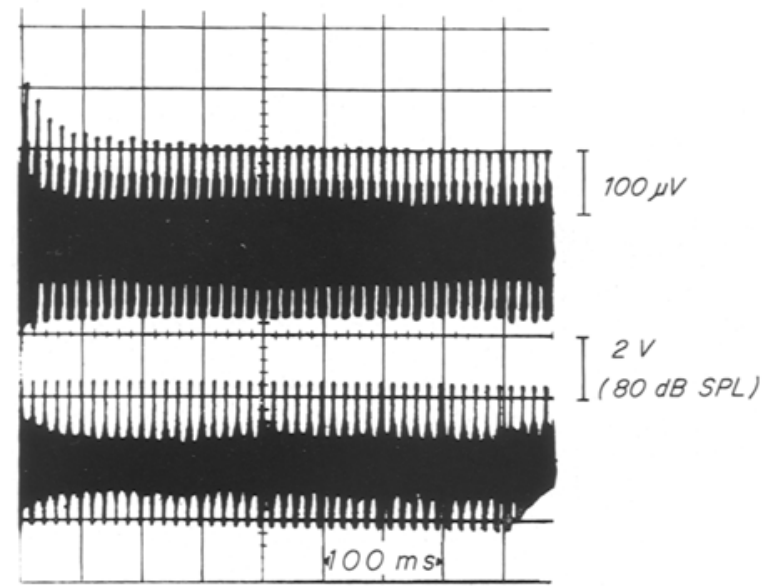

Abb. 7: Hüllkurve der Summenaktionspotentiale des runden Fensters der Katze. Das Einstellen von einem hohen, ersten Potentialwert (initial spike) auf die niedrigeren adaptierten Potentialwerte (steady state amplitude) ist in der oberen Spur deutlich zu erkennen. Die untere Spur zeigt den mit einem Sondermikrophon unmittelbar vor dem Trommelfell gemessenen Schalldrudkverlauf. (Nach Stange, SPRENG \& KeIDEL 1964)

In Abbildung 8 sind die Ergebnisse der quantitativen Untersuchung aufgetragen. Wir betrachten zunächst das erste der als Adaptationsfächer bezeichneten Diagramme (ganz rechts). Hierbei ist die Reizstärke als Abschwächung auf der Abszisse und die Größe der Erregung als Nervenaktionspotential in Mikrovolt auf der Ordinate aufgetragen. Bei einer Kennlinie dieser Art ist zunächst die triviale Beziehung zu erkennen, daß mit abnehmender Reizstärke die Amplitude des Summenaktionspotentials in bestimmter Weise abnimmt. Abhängig jedoch von der Folgefrequenz des Rechteckreizes, also vom jeweiligen Adaptationszustand der Sinneszellen, wird diese Kennlinie zunehmend flacher, wie die verschiedenen eingetragenen Kennlinien andeuten. Sie sind von oben nach unten jeweils einer höheren Reizfrequenz zuzuordnen. Bei einer bestimmten Reizstärke, beispielsweise bei einer Abschwächung von - $40 \mathrm{~dB}$, ist im nichtadaptierten Zustand ein Summenaktionspotential von etwa $300 \mu \mathrm{V}$ zu erwarten. Der Gleichgewichtsendwert bei einer Folgefrequenz von beispielsweise 200/sec (punktiert) beträgt dann nur etwa $150 \mu \mathrm{V}$, der Gleichgewichtsendwert bei einer höheren Folgefrequenz, beispielsweise $1000 / \mathrm{sec}$, ist kleiner und beträgt nur $100 \mu \mathrm{V}$ (offene Kreise). Wir können also durch diese Abbildung den Einfluß der Adaptation auf die ReizstärkeErregungskennlinien der Sinneszellen ablesen.

Die gezeigte Versuchsreihe, welche eine Veränderung dieser sogenannten Adaptationsfächer mit wachsender Streptomycindosis herbeiführt, hatte ursprünglich den Zweck, die selektive Schädigung der Sinneszellen des Gehörs bei Streptomycingaben, 
wie sie dem Kliniker bekannt sind, näher zu klären. Abbildung 8 zeigt mit wachsender Dosis eine charakteristische Veränderung der Form des zweiten Fächers; im Gegensatz zum ersten Fächer (ohne Schädigung) ist dieser sehr stark aufgefächert. Eine Steigerung

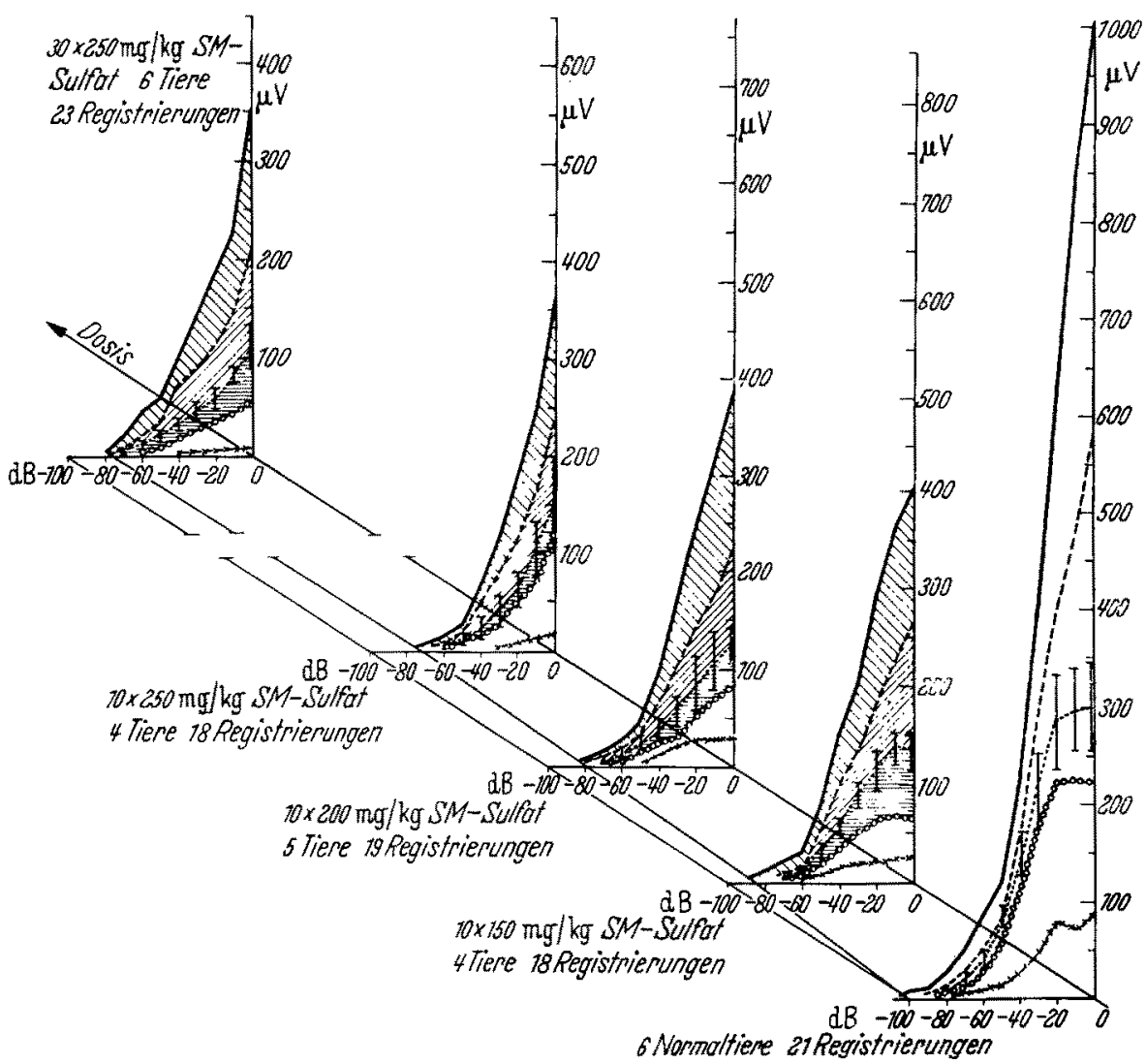

Abb. 8: Adaptationsfächer der Summenaktionspotentiale des Nervus acusticus der Katze bei unbehandelten Kontrolltieren (ganz rechts) und bei mit steigender täglicher Streptomycinsulfatdosis (SM-Sulfat) behandelten Tiere. Die A bs z is se gibt jeweils die Abschwächung des Schalldruckpegels, die Ordinate die Größe der gemessenen Potentiale; die dritte Koordinate gibt die steigende Dosis. Parameter sind die verschiedenen Reizfolgefrequenzen, und zwar pro Fächer von oben nach unten $1 / \mathrm{sec}, 50 / \mathrm{sec}, 200 / \mathrm{sec}, 1000 / \mathrm{sec}, 5000 / \mathrm{sec}$. Die Längsbalken bedeuten $1 \sigma$. (Nach STANGE, SPRENG \& KeIDEL 1964)

der Streptomycinsulfatdosis bringt, wie am dritten Fächer ersichtlich, wieder ein $\mathrm{Zu}$ sammenrücken der Kennlinien. Dieses Zusammendrängen wird mit zunehmender Dosis deutlicher (vierter Fächer). Bei sehr hohen Dosen allerdings (fünfter Fächer) ist wiederum ein Auseinanderstreben $\mathrm{zu}$ beobachten (Signifikanzgrenzen jeweils eingezeichnet).

Was ist die Ursache für diese Anderung des Adaptationsverhaltens? Legt man die RaNKEsche Hypothese einer einfachen, zeitlich verschobenen Oberlagerung eines Bildungs- und eines Rückbildungsstoffwechsels zugrunde, dann zeigt der zweite Fächer 
(nach Streptomycinsulfatgabe) zunächst eine extreme Störung des Bildungsstoffwechsels bei nahezu voll erhaltener Funktionsfähigkeit des Rückbildungsstoffwechsels. Mit anderen Worten: Es tritt anfangs ein geringes Überschießen auf, infolge starker Rückbildung wird jedoch nur ein sehr kleiner Gleichgewichtsendwert erreicht. Zunehmende Streptomycinsulfatdosierung schädigt nun auch den Rïckbildungsstoffwechsel (die Rückbildung wird schwächer, der Gleichgewichtsendwert größer); der Fächer rückt immer näher zusammen. Das Auseinanderrücken des Fächers bei einer relativ hohen Dosierung ist mit einer kompensatorischen Erholung des Bildungsstoffwechsels bei geschädigter Rückbildung ohne weiteres hypothetisch zu erklären.

\section{ENERGIE- UND STOFFWECHSEL}

Diese Befunde, welche zweifellos noch eine zusätzliche experimentelle Stützung benötigen, bringen außerordentlich gewichtige Argumente für die Beteiligung von Stoff wechselvorgängen an der Adaptation der Sinneszellen im Ohr. Eine Deutung der gezeigten progressiven Anderungen in Abhängigkeit von der Streptomycinsulfatdosis ist schwer zu geben, wenn man Stoffwechselvorgänge dieser Art nicht in Betracht zieht, sondern nur Ionenvorgänge an bestimmten Membranen berücksichtigt (HENSEL 1952).

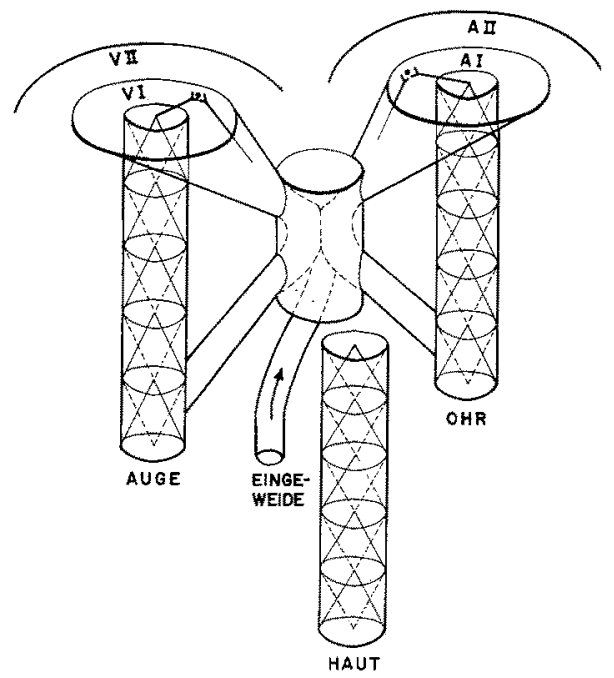

Abb. 9: Wirkungsgefüge der Optimalisierungsfunktion des ZNS, bestehend aus der Vermaschung der einzelnen Sinnesorgansysteme in spezifische und unspezifische Bahn. (Nadh KEIDEL 1963c)

Abbildung 9 führt uns vom Rezeptor wiederum zu zentralnervöseren Stationen der Sinnesorgansysteme; hier ist in einer halbanatomischen Darstellung wieder das Sinnesorgansystem Ohr im Zusammenspiel mit den beiden anderen wichtigen Sinnesorgansystemen aufgezeichnet. Aufsteigend sind die drei Sinneskanäle angedeutet: Auge, Ohr, Haut. In diesem Bild ist die Verzweigung über die Formatio reticularis bemer- 
kenswert, wie sie hier schematisch eingetragen ist. Diese Verzweigung ist allen Sinneskanälen gemeinsam, so daß die Sinneskanäle, wie bereits erwähnt, über die Formatio reticularis miteinander in Verbindung stehen.

Wir erinnern uns, daß die extreme Informationsreduktion durch eine Optimalisierungsfunktion bewerkstelligt wird, die ihren Sitz wahrscheinlich in der Formatio reticularis hat. Drosselungseinwirkungen können nun einmal über die descendierenden

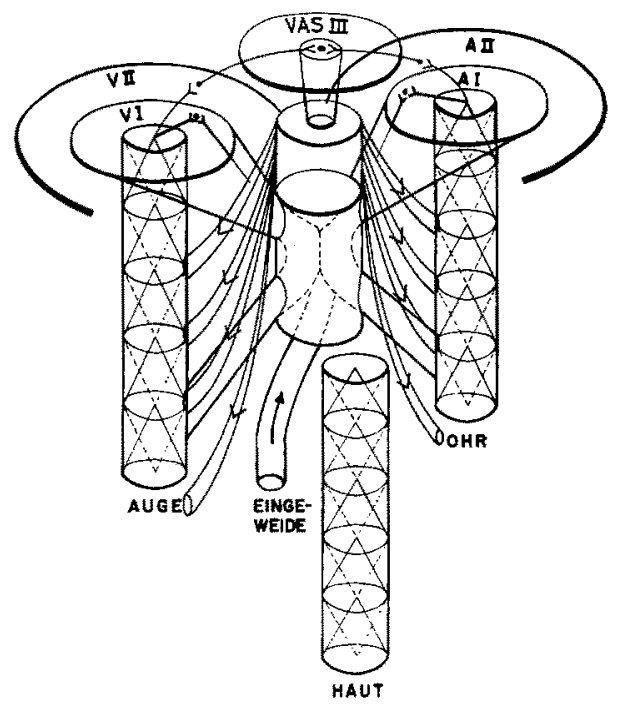

Abb. 10: Wirkungsgefüge der Optimalisierungsfunktion des ZNS, bestehend aus der Vermaschung der einzelnen Sinnesorgansysteme in spezifische und unspezifische Bahn; zusätzlich sind die descendierenden Fasersysteme mit der Fähigkeit $\mathrm{zu}$ selektiver Drosselung eingezeichnet. (Nach KeIDEL 1963c)

Fasersysteme (Abb. 10) an hierarchisch tiefer liegenden Schaltelementen geführt werden; andererseits ist aber auch eine Beeinflussung der Projektionsrindenareale im Sinne dieser Optimalisierungseinrichtung zu fordern.

Will man diese Einwirkung der Optimalisierungsfunktion elektrophysiologisch näher untersuchen, so muß man in diesem Projektionsrindenareal von einer größeren Neuronenpopulation ableiten. Hierbei ist wesentlich, daß sich der Organismus im Wachzustand befindet. Der Registrierung einer durch Reiz ausgelösten corticalen Erregung am wachen, unverletzten Menschen stehen große Schwierigkeiten entgegen. Die Hauptschwierigkeit bereitet das Vorhandensein der normalen Spontanaktivität des Elektroencephalogramms. Leider hat das normale EEG eine viel größere Amplitude als das durch den Reiz hervorgerufene spezifische elektrophysiologisch registrierbare Potential. Es ist uns am Erlanger Institut schon vor längerer Zeit möglich gewesen, unter Benützung komplizierter elektronischer Auswerteanlagen zu zeigen, daß mit Hilfe eines Mittelungsverfahrens dieses Signalstörverhältnis, welches kleiner als 1 ist, verbessert werden kann (KeIDEL 1963c, SpReng \& KeIDel 1963). Wichtig war allerdings der Nachweis, daß die mit dieser Methodik ermittelten Potentiale tatsächlich 
reizspezifischer Natur sind. Wir konnten für sämtliche Sinnesmodalitäten ReizstärkeErregungsbeziehungen aufstellen, die sich in außerordentlich guter Übereinstimmung mit bekannten psychologischen Untersuchungsergebnissen befinden (KEIDEL \& SPRENG 1965).

Wie eingangs erwähnt, besteht ein Informationsengpaß in höheren zentralnervösen Schichten dergestalt, daß eine beschränkte Informationskapazität von etwa $100 \mathrm{bit} / \mathrm{sec}$ für alle Sinneskanäle zur Verfügung steht. Das heißt nichts anderes, als daß ein Sinneskanal, der im gegebenen Augenblick eine bestimmte Informationskapazität besitzt, dann gedrosselt werden muß, wenn zusätzlich ein anderer Sinneskanal eine vorher nicht vorhandene Information anliefert.
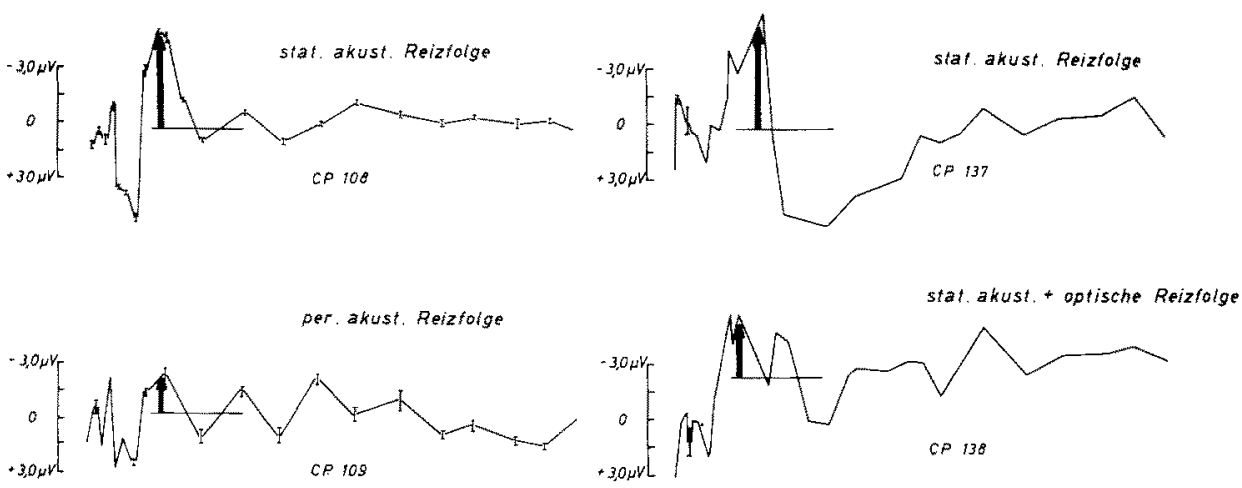

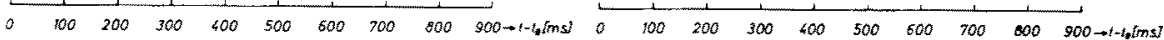

Abb. 11: Der Einfluß der Optimalisierungseinrichtung des ZNS ist mit Hilfe der Registrierung elektronisch gemittelter reizsynchroner langsamer Rindenpotentiale am intakten Sdiädel des Menschen objektivierbar. Nähere Erläuterungen siehe Text. (Nach SPRENG \& KEIDEL 1963)

Abbildung 11 gibt das beim Menschen auf die beschriebene Art und Weise gewonnene Potential als Ausdruck reizsynchroner corticaler Erregungsänderungen wieder. Auf der rechten Bildhälfte erkennt man in der oberen Reihe ein komplexes Potential einer bestimmten Amplitude (Pfeil). Dieses Potential stellt die Antwort auf eine akustische Reizfolge (kurze Sinustöne, beispielsweise $1 \mathrm{sec}$ Dauer, mit statistisch wechselnden Pausen) dar. Gibt man nun die gleiche akustische Reizfolge und mißt dazu wieder das reizsynchrone Potential, blendet jedoch völlig unkorreliert dazu optische Reize (Lichtblitze) ein, dann resultiert das in der unteren Reihe dargestellte Potential; eine deutliche Amplitudenminderung ist zu erkennen. Genau diese Drosselung ist im Sinne der eingangs erwähnten Optimalisierungsfunktion zu fordern: Ein Sinneskanal, in diesem Falle der akustische, wird, obwohl er die gleichen Reize erhält, gedrosselt, wenn gleichzeitig ein anderer Sinneskanal, in diesem Falle der optische Sinneskanal, beansprucht wird.

Das gleiche ist der Fall, wenn man keinen zweiten Sinneskanal beansprucht, sondern im gleichen Sinneskanal den Informationsgehalt des Ereignisses ändert. In Abbildung 11 (links) wird eine statistisch akustische Reizfolge und im Vergleich dazu eine 
periodische Reizfolge angeboten. In der oberen Reihe tritt eine relativ große, in der unteren eine relativ kleine Amplitude des komplexen Potentials auf, das heißt, eine Drosselung erfolgt auch dann, wenn im gleichen Sinneskanal trotz vorhandener Reizung keine neue Information angeboten wird. Die periodische Reizfolge bietet dem Zentralnervensystem die Möglichkeit, innerhalb kurzer Zeit ein Vorhersagemodell aufzubauen. Sofern-keine Anderung in der Reizfolge eintritt, kann also dieser Kanal zugunsten anderer gedrosselt werden (KeIDEL 1963b).

Es handelt sich hier ohne Zweifel um drosselnde Vorgänge zumindest in Höhe der Projektionsrinde, wenn nicht sogar in höheren Hirnrindenarealen sekundärer und tertiärer Art. Ahnlich wie im ausführlich geschilderten Fall der Adaptation im Rezeptor liegen auch hier wieder zwei Möglichkeiten der Wirkungsgradverstellung vor. Einmal ist daran zu denken, daß drosselnde Wirkung durch rein elektrotonische Beeinflussung dieser corticalen Schichten (Neurone) zustande kommt. Diese Ansicht scheinen Untersuchungen von CASPERS (1960) zu unterstützen, der im Tierversuch zeigen konnte, daß Potentiale dieser Art durch eine auf die Hirnrinde aufgebrachte Gleichspannung sehr stark veränderlich sind. Auf der anderen Seite ist an einen wirkungsgradverstellenden Einfluß bestimmter Stoffwechselvorgänge zu denken; in dieser Richtung liegen jedoch praktisch noch keine Untersuchungsbefunde vor.

\section{ZUSAMMENFASSUNG}

1. Durch die Gleichberechtigung der Begriffe "Energie" und "Information" konnten in den letzten Jahrzehnten vor allem in der Sinnesphysiologie entscheidende Fortschritte dadurch erzielt werden, daß unter Verzicht auf die Untersuchung einzelner Energieprozesse zunächst der Informationsablauf im Gesamtfunktionssystem analysiert wurde. Dies wird am Beispiel der Optimalisierungsfunktion des Zentralnervensystems erläutert.

2. Die Adaptation der Sinneszelle stellt scheinbar einen Erregungsverlust, hinsichtlich der Informationsverarbeitung jedoch einen Gewinn dar. Letzterer besteht in der Anpassung eines Bereiches hoher Unterschiedsempfindlichkeit an die mittlere Reizstärke. Elektrophysiologische Untersuchungen am Sinnesorgansystem Ohr weisen auf die Mitbeteiligung und die große Bedeutung des Zusammenspiels mehrerer Stoffwechselvorgänge im Rahmen dieser Adaptation hin.

3. Komplizierte Stoffwechseleinflüsse sind auch bei zentralen Informationsverarbeitungsaufgaben, und zwar speziell bei der Drosselung aktiver Elemente im Zuge der Optimalisierungsfunktion, zu vermuten.

4. Die extreme Informationsreduktion im menschlichen Sensorium beruht auf selektiven Drosselungsvorgängen an hierarchisch tieferen Schichten des Sinnesorgansystems, vor allem auch in Höhe der Hirnrinde. Sie wird durch rückläufige Fasersysteme bewirkt und ist objektivierbar. 


\section{ZITIERTE LITERATUR}

Caspers, H., 1960. Die Beeinflussung der corticalen Gleichspannung durch sensible und sensorische Reize beim wachen, freibeweglichen Tier. Pfïgers Arch. ges. Physiol. 272, 53-54.

Davis, H., 1961. Some principles of sensory receptor action. Physiol. Rev. 41, 391-416.

HeNsel, H., 1952. Physiologie der Thermoreception. Ergebn. Physiol. 47, 166-368.

KeIdeL, W. D., 1961a. Rankes Adaptationstheorie. Z. Biol. 112, 411-425.

- 1961b. Grundprinzipien der akustischen und taktilen Informationsverarbeitung. Ergebn. Biol. 24, 213-246.

- Keidel, U. O. \& Wrgand, M. E., 1961. Adaptation, loss or gain of sensory information. In: International symposium on principles of sensory communication, Mass. Inst. of Technology, 1959. Ed. by W. A. Rosenblith. Wiley, New York, 319-338.

- 1963a. Kybernetische Systeme des menschlichen Organismus. Veröff. ArbGemein. Forsch. NRhein-Westf. 118, 31-71.

- 1963b. Beispiele und Probleme einer kybernetischen Physiologie des ZNS und der Sinne. In: Berichte über den 23. Kongreß der Dt. Ges. für Psychologie. Hogrefe, Göttingen, 103-123.

- 1963c. Tuning between central auditory pathway and the ear. IRE Trans. milit. Electron. 7 (Nr. 283), 131-143.

- 1965. Anatomie und Elektrophysiologie der zentralen akustischen Bahnen. In: Hals-NasenOhren-Heilkunde. Ein kurzgef. Handbuch. Hrsg. von J. Berendes, R. Link \& F. Zöllner. Thieme, Stuttgart, Bd 3.

- \& Spreng, M., 1965. Neurophysiological evidence for the Stevens power function in man. J. acoust. Soc. Am. 38, 191-195.

KÜPFMÜLLER, K., 1959. Informationsverarbeitung durch den Menschen. Nachrtech. Z. 12, 68-74.

MacNicol, Jr., E. F., 1956. Visual receptors as biological transducers. In: Molecular structure and functional activity of nerve cells. A symposium. Ed. by R. G. Grenell \& L. J. Mullins. Am. Inst. of Biol. Sci., Washington, DC.

Magoun, H. W., 1954. Brain stem and higher centers. In: Transactions of the 5th Conference on nerve impulse. Ed. by D. Nachmansohn \& H. H. Merritt. Macy, New York, 11-93.

Miller, G. A., 1956. The magical number seven, plus or minus two. Some limits on our capacity for processing information. Psychol. Rev. 63, 81-87.

MountCAsTLE, V. B., 1959. Convergence and organizational principle in the nervous system. In: International symposium on principles of sensory communication, Mass. Inst. of Technology, 1959. Ed. by W. A. Rosenblith. Wiley, New York (1961).

Ranke, O. F., 1962. Allgemeine Physiologie der Sinnesorgane. In: Handbuch der gesamten Arbeitsmedizin. Hrsg. von E. W. Baader unter Mitw. von G. Lehmann (u. a.). Urban \& Schwarzenberg, München, Bd 1, 207-261.

Shannon, C. E. \& WEAver, W., 1949. The mathematical theory of communication. Univ. of Illinois pr., Urbana, 117 pp.

SPRENG, M., 1964. Die Codierung und Informationsleitung im Zentralnervensystem. In: Kybernetik. Brücke zwischen den Wissenschaften. Hrsg. von H. Frank. 4. Aufl. UmschauVerl., Frankfurt a. M., 73-82.

- \& KEIDEL, W. D., 1963. Neue Möglichkeiten der Untersuchung menschlicher Informationsverarbeitung. Kybernetik 1, 243-249.

Stange, G., SPreng, M. \& Keidel, U. O., 1964. Die Wirkung von Streptomycinsulfat auf Erregung und Adaptation der Haarzellen des Cortischen Organs. Pfiügers Arch. ges. Physiol. 279, 99-120. 


\section{Diskussion im Anschluß an den Vortrag KeDDel \& SPRENG}

KNöTIG: Wie wurden die gezeigten Potentiale in akustisch und optisch hervorgerufene getrennt? SPRENG: Die gezeigten Potentiale wurden durch Mittlung reizsynchroner EEG-Abschnitte gewonnen. Nach einer gewissen Anzahl gleichartiger Reize wurden die Potentiale von der verwendeten Verarbeitungsanlage in der gezeigten Form ausgegeben. Bei Verwendung großflächiger Elektroden ist zweifellos bei g le i ch zeitig e r akustischer und optischer Reizung eine Überlagerung der evozierten Potentiale gegeben. Trotzdem könnte man in diesem Falle durch die verschiedenen Latenzzeiten der optischen und akustischen Komponenten eine Trennung durchführen. Außerdem wäre es möglich, durch eine Lageveränderung kleinerer Elektroden eine Trennung optischer und akustischer Potentiale zu bewirken. In den gezeigten Beispiel jedoch ist eine solche Trennung nicht notwendig, denn die Einsätze des optischen und akustischen Reizes fallen ja überhaupt nicht zusammen. Die optischen Reize treffen völlig unkorreliert zwischen den Pausen zweier akustischer Reize ein und lösen keinen Mittelungsvorgang aus.

ZERBST: Gilt das Massenwirkungsgesetz von GuldBERG \& WAAGE auch für den Rezeptorstoffwechsel in einem offenen System? Wäre es in diesem Falle nicht etwas spezieller zu formulieren? Sie stellen nach der Theorie von RANKe die Erregung proportional zu einer Umsetzgeschwindigkeit dar. Wie sind die Rezeptorpotentiale mit der Umsatzrate gekoppelt? Nach Ihren Versuchen mit Streptomycinsulfat soll allein über Stoffwechselvorgänge das Verhalten der Potentiale zu erklären sein, die Ionenverteilung an den Membranen ist von Stoffwechselvorgängen abhängig. Die Ionenvorgänge an der Membran könnten über Wirksubstanzen in einer nicht bekannten Form beeinflußt werden.

SPRENC: Ihre Einwände sind berechtigt und interessant. Bei den vorgetragenen Versuchen hat uns zunächst nur der elektrophysiologische Nachweis von Stoff wechselvorgängen, insbesondere von mehr als einem Stoffwechselvorgang im Ohr, überhaupt interessiert. Die Darstellung unserer Befunde mit anderen einfachen Modellvorstellungen als auch die Gültigkeit des Massenwirkungsgesetzes für den Rezeptorstoff wedhsel. in einem offenen System haben wir nicht geprïft. Auch zu Ihrer Frage nach der Koppelung der Generatorpotentiale mit der Bildungsgeschwindigkeit beziehungsweise der Geschwindigkeit der Konzentrationsänderung können keine Befunde vorgelegt werden, da die Messung des Generatorpotentials am Ohr nicht möglich ist. Uns hat weniger das Generatorpotential als solches interessiert, als vielmehr die für die Informationsverarbeitung wichtige Umsetzung des mechanischen Reizes an der Haarinsertionsstelle der Sinneszelle in die Erregung des abführenden Nerven.

Hess: Man muß heute auch die Aktivierung von Enzymen in Betracht ziehen, die RankE noch unbekannt waren. Es gibt Enzyme, die neuronenartig Metaboliten des Stoffwechsels um das 500- bis 1000 fache aktivieren. Mit dieser Erkenntnis sind die Arbeiten von RaNKE sehr interessant geworden.

Detchev: Die Hypothese von Ranke gestattet auch eine andere Deutung. Man kann die Wirkung als eine Veränderung des Eigenstoffwechsels ansehen. Die Effekte, die man hier dem Wandler zuschrieb, haben wir auch an Einzelzellen von Nitella und an Pflanzen erhalten. Stärkere in Zeitabständen erfolgende Reize können einen normalen Reizeffekt verursachen. Eine Drosselung erhielten wir, wenn wir Blätter mit Lidht und zuvor mit Temperatur gereizt hatten. In diesen Systemen sind keine intermembranellen Zusammenhänge anzunehmen.

SPRENG: Diese vorgetragene Deutung ist sehr interessant. Jedoch miißte vor einer Ubertragung auf die beschriebenen Adaptationszeitgänge festgestellt werden, ob die Vorgänge an Pflanzenzellen in ähnlich extrem kurzen Zeitbereichen ablaufen können. 